\title{
TIPS improves outcomes in patients with HCC and symptomatic portal hypertension: a multi-institution experience
}

\author{
Huzheng Yan ${ }^{1,2 \dagger}$, Zhenkang Qiu ${ }^{2 \dagger}$, Zhanwang Xiang ${ }^{1 \dagger}$, Kai Feng ${ }^{3}$, Mingsheng Huang ${ }^{1 * \dagger}$ and Fei Gao ${ }^{2^{* \dagger}}$
}

\begin{abstract}
Background: Hepatocellular carcinoma (HCC) with symptomatic portal hypertension (SPH) has poor prognosis. A transjugular intrahepatic portosystemic shunt (TIPS) relieves SPH, but its application in HCC remains unclear. We evaluated TIPS efficacy in patients with HCC and SPH.

Methods: Pre- and post-TIPS Child-pugh(C-P) scores and stages in $123 \mathrm{HCC}$ patients with SPH from three centers were compared. The impact of postoperative C-P stage indicators on overall survival (OS) was explored.

Results: Post-TIPS responses to SPH included complete response (CR) (92 [74.8\%]), partial response (PR) (23 [18.7\%]), and nonresponse (NR) (8 [6.5\%]). The control (proportion of CR and PR) for SPH was 93.5\%. Median C-P scores pre-TIPS and at one month post-TIPS were 8 (IQR 6-9) and 7 (IQR 6-8), respectively $(P<0.001)$. Forty-one (33.3\%) patients had C-P downstaging; 73 (59.3\%) had lowered C-P scores; and 73 (59.3\%) received intrahepatic local therapy post-TIPS. The median OS was 10.7 (1.1-55.2) months. Among the five indicators of C-P stage, lower post-TIPS ascites grading $[(0 / 1) /(2 / 3) ; P=0.014, \mathrm{HR}=0.31$ (95\% Cl: $0.12-0.79)]$ and bilirubin $[<34 / \geq 34 \mu \mathrm{mol} / \mathrm{L} ; P=0.022, \mathrm{HR}=0.47$ (95\% Cl: $0.23-0.82)]$ and prothrombin time prolongation $<6 \mathrm{~s}[<6 / \geq 6 \mathrm{~s} ; P=0.001, \mathrm{HR}=0.17$ (95\% Cl: 0.06-0.47)] were independent protective indicators of OS. These three indicators were included in the nomogram model to predict survival probabilities.
\end{abstract}

Conclusions: TIPS is safe and effective for HCC with SPH. This procedure can relieve the symptoms, enable subsequent antitumor therapy, and bring survival benefits, possibly through improved liver function by reducing C-P stage.

Keywords: Hepatocellular carcinoma, Portal hypertension, Child-Pugh stage, TIPS

*Correspondence: huangmsh@mail.sysu.edu.cn; gaof@sysucc.org.cn ${ }^{\dagger}$ Huzheng Yan, Zhenkang Qiu, Zhanwang Xiang, Mingsheng Huang and Fei Gao contributed equally to this work.

1 Department of Interventional Radiology, The Third Affiliated Hospital of Sun Yat-Sen University, 600 Tianhe Road, Guangzhou 510630, China ${ }^{2}$ Department of Minimally Invasive \& Interventional Radiology, State Key Laboratory of Oncology in South China, Collaborative Innovation Center for Cancer Medicine, Sun Yat-Sen University Cancer Center, 651 Dongfeng Road East, Guangzhou 510060, China

Full list of author information is available at the end of the article

\section{Introduction}

Eighty percent of patients with hepatocellular carcinoma (HCC) have end-stage cirrhosis [1, 2]. Liver cirrhosisrelated portal hypertension leads to serious complications such as refractory ascites and upper gastrointestinal bleeding, which exacerbate the liver function burden and lead to a poor prognosis [3, 4]. Most patients with HCC have symptomatic portal hypertension (SPH) [5, 6], which may disrupt tumor treatment and threaten survival prognosis $[2,3,7]$.

The emergence of molecular-targeted drugs and immune checkpoint inhibitors has greatly improved 
survival rates of advanced $\mathrm{HCC}[8,9]$. However, the presence of SPH with $\mathrm{HCC}$ leads to more conservative treatment [2, 3]. Portal hypertension is an independent prognostic factor that increases 3- and 5-year mortality rates [10]. The European Association for the Study of the Liver recommends that patients with resectable $\mathrm{HCC}$ and clinically significant portal hypertension be evaluated circumspectly for appropriate treatment $[2,11,12]$. For patients with unresectable advanced $\mathrm{HCC}$ with $\mathrm{SPH}$, there is currently no specific guideline to define the treatment strategy.

A transjugular intrahepatic portosystemic shunt (TIPS) has been recommended for managing SPH in end-stage liver disease [13]. Most studies have shown that TIPS can effectively control ascites, reduce the risk of esophagogastric vein rupture and re-bleeding, and improve liver function [3, 14]. The Child-Pugh (C-P) stage is most commonly used to evaluate liver function and prognosis in patients with cirrhosis. Its limitations have been described in detail [15]. For HCC patients with $\mathrm{SPH}$, the liver function is more likely to be determined by the status of the tumor and the non-tumor liver. Specifically, the ascites and plasma albumin levels in the $\mathrm{C}-\mathrm{P}$ stage are interrelated, and bleeding caused by portal hypertension seriously affects the C-P stage, while TIPS can improve the bleeding caused by portal hypertension. Therefore, we have to consider whether the $\mathrm{C}-\mathrm{P}$ stage after TIPS represents the true prognosis of a patient. It is unknown whether the C-P stage is appropriate for $\mathrm{HCC}$ with $\mathrm{SPH}$, and no research has clarified which of its five indicators is most likely to be improved after TIPS. With improved stent technology, some small studies have confirmed the feasibility and efficacy of TIPS in patients with HCC [16, 17]. However, little existing literature has reported overall survival (OS) related to TIPS in patients with HCC with $\mathrm{SPH}$. The purpose of our study was to evaluate the efficacy of TIPS in HCC patients with SPH and to explore post-TIPS prognostic factors using an improved visualization nomogram model based on the C-P stage.

\section{Methods}

\section{Study population}

A total of 3911 patients with HCC from three interventional radiology centers from January 2016 to January 2020 were evaluated retrospectively. Inclusion criteria were as follows: diagnosed with HCC complicated by $\mathrm{SPH}$, received TIPS, and had a tumor volume no more than $70 \%$ of the liver volume. Excluded patients had primary cholangiocarcinoma, multiple hepatic cysts, refractory biliary and pancreatic obstruction, liver failure, or severe cardiopulmonary dysfunction. Finally, 123 HCC patients with SPH who had received TIPS were included in this study. The Ethics Committee of the Sun
Yat-sen University Cancer Center approved the study and waived the requirement for informed consent because as a retrospective study, this study did not interfere with patients' treatment choices and thus was a very low risk to patients.

$\mathrm{SPH}$ in all of the patients manifested as refractory ascites (RA) (51 [41.5\%]) and variceal bleeding (72 [58.5\%]). Five cases (4.1\%) had severe diarrhea. In terms of variceal bleeding, $15(20.8 \%)$ patients had active variceal bleeding after endoscopic or medical treatment failure, and 57 (79.2\%) patients underwent prevention for variceal re-bleeding.

\section{Definitions}

The HCC diagnosis was based on the history of hepatitis, alpha-fetoprotein (AFP) levels, and imaging. SPH was defined as the presence of RA or variceal bleeding more than once. RA was classified into mild, moderate, or large/gross ascites in accordance with the International Club of Ascites definitions [3].

Responses to TIPS were classified as follows: complete response $(\mathrm{CR})$, where no further variceal bleeding occurred and there was no clinically detectable ascites with or without a diuretic or salt-restricted diet; partial response (PR), where there was a small amount of ascites not requiring special paracentesis; and nonresponse (NR), where there was a large amount of ascites needing special intervention or there was recurrence of variceal bleeding [16].

Color Doppler ultrasonography (CDUS) was used to assess shunt patency. Shunt dysfunction was defined as a maximum flow velocity $>180 \mathrm{~cm} / \mathrm{s}$ or $<60 \mathrm{~cm} / \mathrm{s}$ in the shunt or $<30 \mathrm{~cm} / \mathrm{s}$ in the main portal vein and a large amount of ascites or variceal re-bleeding. Suspected shunt dysfunction was confirmed with portal angiography showing a portosystemic pressure gradient $(\mathrm{PPG}) \geq 15 \mathrm{~mm} \mathrm{Hg}[16,17]$.

\section{TIPS procedure}

As previously described [16, 17], the TIPS procedure involved establishing an artificial shunt channel in the hepatic vein and the main portal vein or left or right branches through the jugular vein approach and implanting the shunt with a stent. All of the study patients received covered stent (Viatorr; W. L. Gore \& Associates, Inc., Flagstaff, AZ, USA) implantation, and 27 (22.0\%) received an additional bare stent (E-Luminexx; Bard Medical Division, Covington, GA, USA) implantation to ensure shunt function. The stent diameter was $8 \mathrm{~mm}$, and the length was either 6,8 , or $10 \mathrm{~cm}$. Portal and vena cava pressures were measured to calculate the PPG preand post-TIPS. Anticoagulant or diuretic treatments were administered post-TIPS as needed. Eighty-two 
(66.7\%) patients received $10-20 \mathrm{mg} /$ day oral rivaroxaban (Xarelto Fine Granules, Bayer, Leverkusen, Germany), and $74(60.2 \%)$ patients received diuretic treatment or a salt-limited diet.

\section{Follow-up}

All of the patients were followed up with until death or until the last contact, with primary end points being OS and effectiveness. The main observation indicators were $\mathrm{C}-\mathrm{P}$ stage, $\mathrm{C}-\mathrm{P}$ scores pre- and post-TIPS, responses to TIPS, and complications. The median follow-up time was 13 months. Laboratory tests of hematology, liver, kidney, and coagulation functions were performed every 1-2 weeks and then monthly. Abdominal CT/MR and CDUS were performed at weeks 1,2 , and 3 and then every two months. Examination intervals were shortened when symptoms recurred or as necessary.

\section{Statistical analysis}

Statistical analysis was performed in SPSS software (IBM, Chicago, USA). All tests were two-tailed, and $P<0.05$ was considered a significant difference. Continuous variables were presented using the median and interquartile range (IQR) values. We used the Pearson chi-square test to compare qualitative data and Student's $t$ test for quantitative data. Kaplan-Meier analysis (log-rank test) was used for subgroup survival analysis. The impact of the five indicators of postoperative $\mathrm{C}-\mathrm{P}$ stage on OS was explored. The independent prognostic factors were selected to constitute the nomogram for prediction of survival probability.

\section{Results}

\section{Patients' characteristics pre-TIPS}

A total of 123 patients were included in the study (Table 1). The median age was 58 (47-64) years, with 112 (91.1\%) men and 11 (8.9\%) women. A total of 117 (95.2\%) patients had a history of hepatitis $\mathrm{B}$. The median MELD was 14.5 (13.34-15.75). The distribution of Barcelona Clinic Liver Cancer (BCLC) classification stages A, B, $\mathrm{C}$, and D was 14 (11.4\%), 27 (22.0\%), 71 (57.7\%), and 11 (8.9\%), respectively. Eighty-one (65.9\%) patients had AFP levels $\geq 100 \mathrm{ng} / \mathrm{mL} ; 81$ (65.9\%) had multiple tumors; 80 (65.0\%) had portal vein tumor thrombosis (PVTT).

\section{Antitumor therapy}

One month post-TIPS, according to the SPH condition, liver function, and tumor stage, patients were reassessed with $\mathrm{C}-\mathrm{P}$ stage and scores to make a decision about appropriate intrahepatic local treatment or systemic treatment. Intrahepatic local treatments included mainly transarterial chemoembolization (TACE) and microwave ablation (MWA), while systemic treatments included
Table 1 Patient characteristics

\begin{tabular}{|c|c|}
\hline Characteristics & $N(\%) /$ median $\left(\mathrm{IQR}^{\mathrm{a}}\right)$ \\
\hline \multicolumn{2}{|l|}{ Gender } \\
\hline Male & 112(91.1\%) \\
\hline Female & $11(8.9 \%)$ \\
\hline Age & $58(47-64)$ \\
\hline$<60$ & $69(56.1 \%)$ \\
\hline$\geq 60$ & $54(43.1 \%)$ \\
\hline \multicolumn{2}{|c|}{ Aetiology of liver disease } \\
\hline Hepatitis B & $117(95.2 \%)$ \\
\hline Hepatitis C & $3(2.4 \%)$ \\
\hline Alcoholic cirrhosis & $3(2.4 \%)$ \\
\hline \multicolumn{2}{|l|}{ BCLC classification ${ }^{a}$} \\
\hline A & $14(11.4 \%)$ \\
\hline B & $27(22.0 \%)$ \\
\hline C & $71(57.7 \%)$ \\
\hline D & $11(8.9 \%)$ \\
\hline \multicolumn{2}{|l|}{$\mathrm{PVTT}^{\mathrm{a}}$} \\
\hline No & $43(35.0 \%)$ \\
\hline Yes & $80(65.0 \%)$ \\
\hline \multicolumn{2}{|l|}{ Number of tumors } \\
\hline Single & $42(34.1 \%)$ \\
\hline Multiple & $81(65.9 \%)$ \\
\hline MELD ${ }^{a}$ & $14.50(13.34-15.75)$ \\
\hline$<15$ & $76(61.8 \%)$ \\
\hline$\geq 15$ & $47(38.2 \%)$ \\
\hline \multicolumn{2}{|c|}{ Symptomatic portal hypertension } \\
\hline Refractory ascites & $51(41.5 \%)$ \\
\hline Variceal bleeding & 49(39.8\%) \\
\hline Both & $23(18.7 \%)$ \\
\hline Refractory diarrhea & $5(4.1 \%)$ \\
\hline \multicolumn{2}{|l|}{ Laboratory tests } \\
\hline Platelets [109/L] & $88(65-125)$ \\
\hline$I^{N} R^{a}$ & $1.26(1.15-1.43)$ \\
\hline $\mathrm{AST}[\mathrm{U} / \mathrm{L}]^{\mathrm{a}}$ & $46.3(34.0-65.0)$ \\
\hline $\operatorname{ALT}[U / L]^{a}$ & $37.6(25.4-52.1)$ \\
\hline Albumin $[\mathrm{g} / \mathrm{dL}]$ & $33.2(30.2-36.8)$ \\
\hline Creatinine $[\mathrm{mg} / \mathrm{dL}]$ & $72(62.2-86.5)$ \\
\hline Bilirubin [mg/dL] & $29.8(19.2-41.0)$ \\
\hline $\operatorname{AFP}[n g / m L]^{a}$ & $16.2(4.81-463.78)$ \\
\hline$<100$ & $42(34.1 \%)$ \\
\hline$\geq 100$ & $81(65.9 \%)$ \\
\hline
\end{tabular}

PVTT Portal vein tumor thrombosis, MELD Model of end stage liver disease, INR International normalised ratio, AST Aspartate aminotransferase, ALT Alanine transaminase, AFP Alpha fetoprotein, CI Confidence interval, IQR Interquartile range

a Barcelona Clinic Liver Cancer

sorafenib (Xarelto Fine Granules, Bayer, Leverkusen, Germany), lenvatinib (Eisai Co Ltd, Tokyo, Japan), or apatinib (Hengrui Medicine, Jiangsu, China). Eighteen (14.6\%) and $14(11.4 \%)$ patients received TACE alone 
or MWA alone, respectively, and 41 (33.3\%) patients received TACE combined with MWA. Among 36 (29.3\%) patients who underwent systemic treatment, 11 (30.6\%) received combined TACE or MWA treatment. Finally, 109 (88.6\%) patients received antitumor therapy, and 73 (59.3\%) received intrahepatic local therapy post-TIPS.

\section{Efficacy of TIPS and shunt dysfunction}

The average PPG decreased from pre-TIPS $(29.4 \mathrm{~mm}$ $\mathrm{Hg})$ to post-TIPS $(12.5 \mathrm{~mm} \mathrm{Hg})$. The pre-TIPS level of ascites was graded 0/I and II/III in $56(45.5 \%)$ and 67 (54.5\%) patients, respectively. At one month post-TIPS, ascites was graded 0/I and II/III in 107 (87.0\%) and 16 (13.0\%) patients, respectively. The number of patients with ascites graded II/III decreased $(P<0.001)$. Of the 72 patients with variceal bleeding as the main symptom, only seven $(9.7 \%)$ reported this symptom one year post-TIPS. Overall, the responses of SPH to TIPS included CR (92 [74.8\%]), PR (23 [18.7\%]), and NR (8 [6.5\%]). The control (proportion of CR and PR) for SPH was $93.5 \%$.

Twenty (16.3\%) patients had shunt dysfunction during the follow-up as confirmed by CDUS and enhanced CT. The primary patency rate at 90 days was $95.1 \%(115 / 123)$. The causes of shunt dysfunction included thrombosis $(6[30 \%])$ and/or tumor invasion (14 [70\%]), and 11 cases underwent a TIPS revision via balloon dilation or implantation of another bare stent. Nine patients refused further intervention and chose conservative treatment.

\section{TIPS-related complications}

The most common post-TIPS complications were ALT/ AST $(77[62.6 \%])$ or bilirubin elevation (72 [58.2\%]), which in most cases did not exceed four times the normal value. One case required percutaneous biliary drainage; one case developed multiple organ failure and died perioperatively; and the remaining patients recovered quickly after receiving liver protection treatment. Nine (7.3\%) cases of suspected abdominal bleeding were monitored by continuous blood count and CDUS. Most of the patients recovered after conservative treatment (blood transfusion, hemostatic drugs, vital signs monitoring, etc.). Only one case developed hemorrhagic shock due to puncture injury rather than due to tumor rupture; this patient recovered after receiving interventional embolization for hemostasis and intensive care. Of the $13(10.6 \%)$ patients who developed hepatic encephalopathy (HE) post-TIPS, only one had an HE of grade III/IV with loss of consciousness requiring intensive care treatment, while the remaining 12 patients had HE with grade I/II and received standard medical treatment to control HE.

\section{Changes in C-P Stages and five indicators}

As shown in Table 2, pre-TIPS C-P stage A, B, and C was found in $32(26.0 \%), 79(64.2 \%)$, and $12(9.8 \%)$ patients, respectively. One month after TIPS, there were 56 (45.5\%), 58 (47.2\%), and $9(7.3 \%)$ patients with C-P stages $\mathrm{A}, \mathrm{B}$, and $\mathrm{C}$, respectively. Forty-one (33.3\%) patients had C-P downstaging, whereas in 70 (56.9\%) and 12 (9.8\%) patients, the $\mathrm{C}-\mathrm{P}$ stage remained unchanged and increased, respectively. The post-TIPS $\mathrm{C}-\mathrm{P}$ stage was

Table 2 Changes in the Child-Pugh scores, Child-Pugh stages

\begin{tabular}{|c|c|c|c|}
\hline Variable & Before TIPS & $\begin{array}{l}\text { One month after } \\
\text { TIPS }\end{array}$ & $P$ \\
\hline \multicolumn{4}{|l|}{ Response to TIPS ${ }^{b}$} \\
\hline $\mathrm{CR}$ & & $92(74.8 \%)$ & \\
\hline$P R$ & & $23(18.7 \%)$ & \\
\hline NR & & $8(6.5 \%)$ & \\
\hline Child-Pugh stage & & & $0.006^{*}$ \\
\hline A & $32(26.0 \%)$ & $56(45.5 \%)$ & \\
\hline B & $79(64.2 \%)$ & $58(47.2 \%)$ & \\
\hline C & $12(9.8 \%)$ & $9(7.3 \%)$ & \\
\hline \multicolumn{4}{|c|}{ Change of Child-Pugh stage ${ }^{b}$} \\
\hline Down & & $41(33.3 \%)$ & \\
\hline Unchanged & & $70(56.9 \%)$ & \\
\hline Elevated & & $12(9.8 \%)$ & \\
\hline Child-Pugh scores & $8(6-9)$ & $7(6-8)$ & $<0.001^{* *}$ \\
\hline \multicolumn{4}{|c|}{ Change of Child-Pugh scores ${ }^{\mathrm{b}}$} \\
\hline Down & & $73(59.3 \%)$ & \\
\hline Unchanged & & $28(22.8 \%)$ & \\
\hline Elevated & & $22(17.9 \%)$ & \\
\hline$H^{a}$ & & & $1.000^{*}$ \\
\hline$|/| \mid$ & $4(3.3 \%)$ & $12(9.8 \%)$ & \\
\hline III/IV & 0 & $1(0.8 \%)$ & \\
\hline \multicolumn{4}{|l|}{ Grading of ascites } \\
\hline $0 / 1$ & $56(45.5 \%)$ & $107(87.0 \%)$ & $<0.001^{*}$ \\
\hline $2 / 3$ & $67(54.5 \%)$ & $16(13.0 \%)$ & \\
\hline $\begin{array}{l}\text { Bilirubin(IQR } \\
\text { umol/L) }\end{array}$ & $29.5(19.2-41.0)$ & $37.4(25.6-48.1)$ & $<0.001^{* *}$ \\
\hline$<34$ & 75(61.0\%) & $51(41.4 \%)$ & \\
\hline$\geq 34$ & 48(39.0\%) & $72(58.6 \%)$ & \\
\hline Albumin (g/L) & $33.2(30.2-36.8)$ & $34.7(33.1-36.8)$ & $0.134^{* *}$ \\
\hline$<35$ & $72(58.5 \%)$ & $65(52.8 \%)$ & \\
\hline$\geq 35$ & $51(41.5 \%)$ & $58(47.2 \%)$ & \\
\hline $\mathrm{PT}(\mathrm{s})^{\mathrm{a}}$ & $14.2(13.1-15.6)$ & $15.5(14.1-17.1)$ & $<0.001^{* *}$ \\
\hline Prolonged < 6 & $117(95.1 \%)$ & $71(57.7 \%)$ & \\
\hline Prolonged $\geq 6$ & $6(4.9 \%)$ & $52(42.3 \%)$ & \\
\hline
\end{tabular}

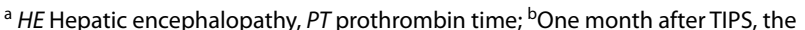
Child-Pugh stage and Child-Pugh score were reassessed. The responses to TIPS: complete response $(C R)$, no further variceal bleeding and having no clinically detectable ascites with or without diuretic or salt-restricted diet; partial response $(P R)$, having a small amount of ascites not requiring special paracentesis; and nonresponse $(N R)$, having a large amount of ascites needing special intervention or variceal bleeding recurrence. ${ }^{*} \mathrm{Chi}$-square test; ${ }^{* *}$ Paired t-test 
lower $(P=0.06)$. The median preoperative and postoperative $\mathrm{C}-\mathrm{P}$ scores were 8 (IQR 6-9) and 7 (IQR 6-8), respectively. Seventy-three (59.3\%) patients had a lowered C-P score, and only $22(17.9 \%)$ had an increased score; post-TIPS C-P scores were lower than pre-TIPS scores $(P<0.001)$.

The incidence of HE post-TIPS (13 [10.6\%]) was not significantly higher than that of pre-TIPS (4 [3.3\%]) $(P=1.000)$. The median pre- and post-TIPS bilirubin level was $29.5 \mu \mathrm{mol} / \mathrm{L}(\mathrm{IQR}, 19.2-41.0)$ and $37.4 \mu \mathrm{mol} / \mathrm{L}$ (IQR, 25.6-48.1), respectively, and prothrombin time (PT) was $14.2 \mathrm{~s}$ (IQR,13.1-15.6) and $15.5 \mathrm{~s}$ (IQR, 14.117.1), respectively. Post-TIPS bilirubin and PT increased $(P<0.001)$. The pre- and post-TIPS grades 2 and 3 of ascites were found in $67(54.5 \%)$ and $16(13.0 \%)$ patients, respectively. Post-TIPS ascites was improved $(P<0.001)$, and albumin levels (34.7 g/L [33.1-36.8]) had an increasing trend $(P=0.134)$ compared with pre-TIPS levels (33.2 g/L [30.2-36.8]).

\section{OS}

The median OS was 10.7 (range, 1.1-55.2) months. The median OS of BCLC A, B, C, and D was 18 (range, 2.651.3), 15.1 (range, 1.6-55.2), 7.8 (range, $1-39.1$ ), and 5.3 (range, 3.6-30.5) months, respectively. The OS of HCC with RA and variceal bleeding was 10.3 (range, 1.1-51.3) and 10.8 (range, 1.2-55.2) months, respectively.

As shown in Table 3 and Fig. 1, pre-TIPS, patients in $\mathrm{C}-\mathrm{P}$ stage $\mathrm{A}$ had a longer OS than those in stage $\mathrm{C}[\mathrm{A} / \mathrm{C}$ : $P=0.005, \mathrm{HR}=0.27$ (95\% CI: 0.11-0.67)], and there was no difference in OS between stages $\mathrm{B}$ and $\mathrm{C}[\mathrm{B} / \mathrm{C}$ : $P=0.335, \mathrm{HR}=0.69$ (95\% CI: 0.32-1.46)]. One month post-TIPS, cases with a lower $\mathrm{C}-\mathrm{P}$ stage $[\mathrm{A} / \mathrm{C}: P<0.001$, $\mathrm{HR}=0.13$ (95\% CI: $0.06-0.28)$; $\mathrm{B} / \mathrm{C}: P<0.001, \mathrm{HR}=0.22$ (95\% CI: 0.10-0.47)] or a C-P stage that did not increase [non-elevated /elevated: $P=0.003, \mathrm{HR}=0.37(95 \% \mathrm{CI}$ : $0.20-0.71)]$ showed better OS. The CR-to-TIPS [CR/ (PR/NR): $P<0.001, \mathrm{HR}=0.38$ (95\% CI: $0.23-0.63$ )] also showed better OS.
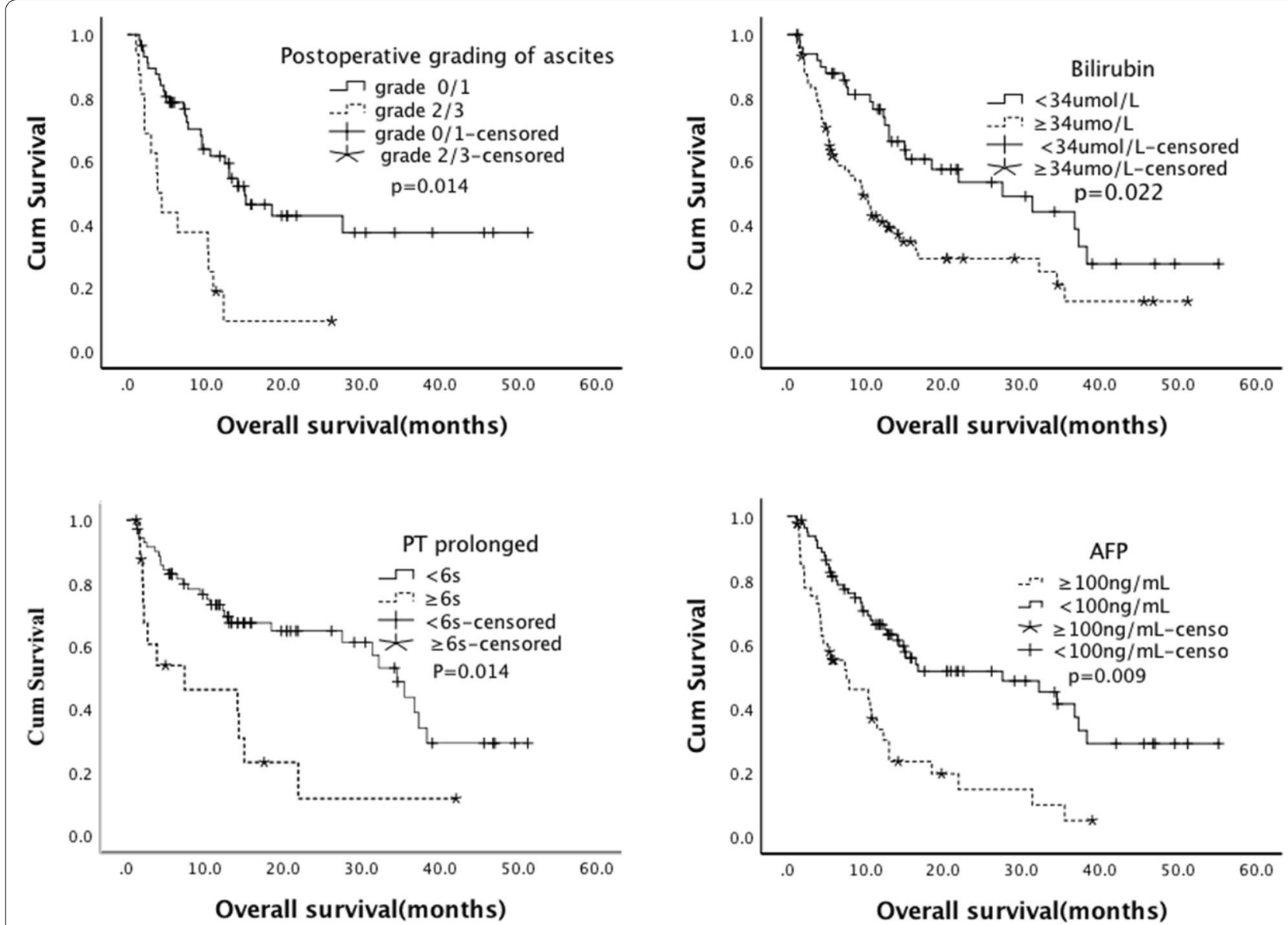

Fig. 1 Overall survival (Kaplan-Meier analysis). a Postoperative Child-Pugh stage. b Bilirubin. c Prolonged prothrombin time (PT). d Alpha-fetoprotein (AFP) 
Table 3 Univariate analysis related to OS

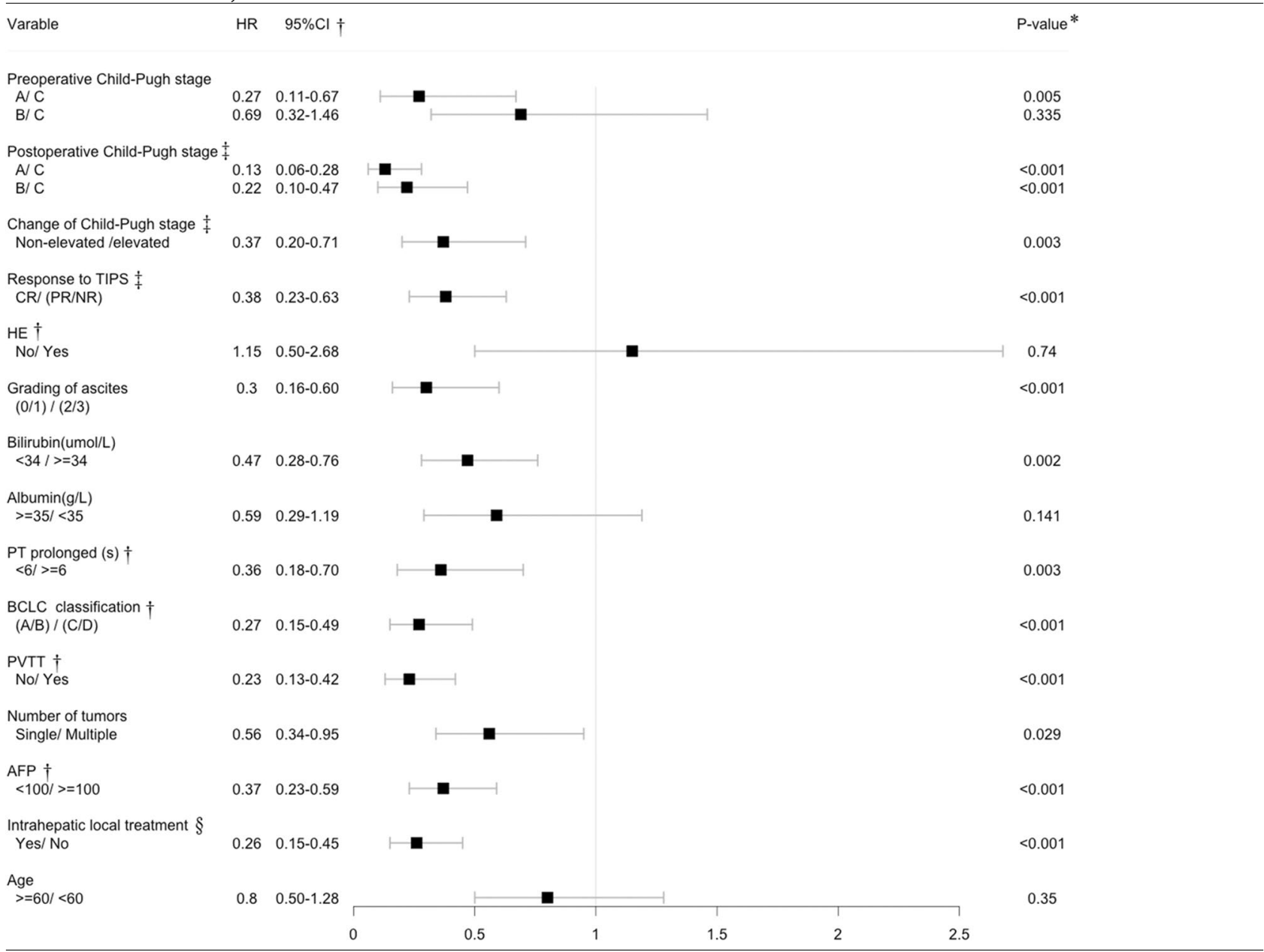

THE Hepatic encephalopathy, PT Prothrombin time, BCLC Barcelona Clinic Liver Cancer, PVTT Portal vein tumor thrombosis, AFP Alpha fetoprotein, CI Confidence interval; ¥One month after TIPS, the Child-Pugh stage and Child-Pugh score were reassessed. The responses to TIPS: complete response (CR), no further variceal bleeding and having no clinically detectable ascites with or without diuretic or salt-restricted diet; partial response $(P R)$, having a small amount of ascites not requiring special paracentesis; and nonresponse $(N R)$, having a large amount of ascites needing special intervention or variceal bleeding recurrence. §lncluding transarterial chemoembolization and ablation. $P^{*}$ Chi-square test

Among the five indicators of post-TIPS C-P stage, good OS was related to lower grading of ascites $[(0 / 1) /(2 / 3)$ : $P<0.001, \mathrm{HR}=0.30(95 \% \mathrm{CI}: 0.16-0.60)$ ], lower level of bilirubin $[<34 / \geq 34 \mu \mathrm{mol} / \mathrm{L}: P=0.002, \mathrm{HR}=0.47(95 \%$ CI: 0.28-0.76)], and PT prolonged for $<6 \mathrm{~s}[<6 / \geq 6 \mathrm{~s}$ : $P=0.003, \mathrm{HR}=0.36$ (95\% CI: 0.18-0.70)]. There was no significant difference in HE or level of albumin $\geq 35$ or $<35 \mathrm{~g} / \mathrm{L}(P>0.05)$. In terms of tumor factors, good OS results were associated with a lower BCLC classification, no PVTT, presence of a single tumor, lower level of AFP, and intrahepatic local treatment.

Table 4 shows the Cox proportional-hazards regression analysis, indicating lower postoperative grade of ascites $[(0 / 1) /(2 / 3): P=0.014, \mathrm{HR}=0.31$ (95\% CI: $0.12-0.79)]$, lower postoperative level of bilirubin $[<34 / \geq 34 \mu \mathrm{mol} / \mathrm{L}$ : $P=0.022, \quad H R=0.47$ (95\% CI: 0.23-0.82)], prolonged post-TIPS $\mathrm{PT}<6$ s $[<6 / \geq 6$ s: $P=0.001, \quad \mathrm{HR}=0.17$
(95\% CI: 0.06-0.47)], and AFP level $<100 \mathrm{ng} / \mathrm{mL}$ $[<100 / \geq 100 \mathrm{ng} / \mathrm{mL}: P=0.009$, HR $=0.31$ (95\% CI: $0.13-$ $0.75)]$ as the independent predictors of better OS.

Based on the statistically significant variables from the multivariate analysis, as shown in Fig. 2, a nomogram was constructed based on the above three variables to predict survival probabilities at $6,12,18,24$, and 36 months.

\section{Discussion}

SPH severely affects the prognosis of patients with HCC and increases the risk of death. Our research demonstrated that TIPS can bring survival benefits, which may occur through the improved liver function. We also proposed a simple model to predict OS based on the improved C-P stage.

There are no treatment guidelines for $\mathrm{HCC}$ with $\mathrm{SPH}$. The few reports on the use of TIPS in HCC have 
Table 4 Cox proportional hazards regression analysis related to OS

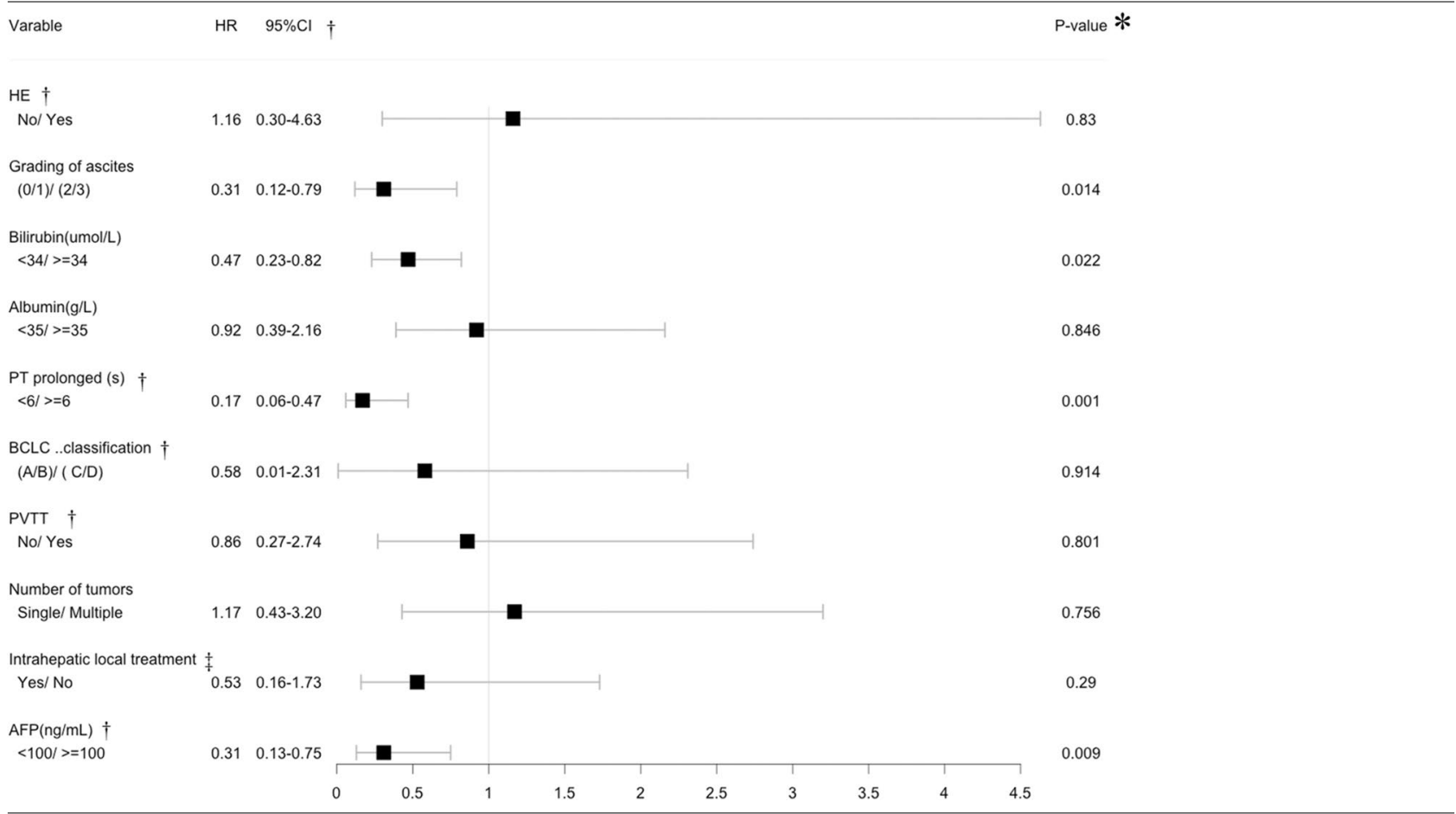

THE Hepatic encephalopathy, PT Prothrombin time, BCLC Barcelona Clinic Liver Cancer, PVTT Portal vein tumor thrombosis, AFP Alpha fetoprotein, CI Confidence interval; ‡.\$Including transarterial chemoembolization and ablation. $P^{*}$ Chi-square test

described its feasibility and safety [16-19]. The OS of $\mathrm{HCC}$ is determined by tumor burden factors, liver function, and complications related to portal hypertension [2, 3, 7]. Complications associated with SPH may be more life-threatening than the tumor burden because SPH often leads to emergency situations [13, 17, 20, 21]. Our results showed that control (proportion of CR and PR) of SPH was achieved in $93.5 \%(115 / 123)$ of patients, and only $6.5 \%(8 / 123)$ of patients showed NR; thus, TIPS was effective for patients with HCC. This high control rate indicates that TIPS can alleviate the symptoms of SPH in patients with $\mathrm{HCC}$ and reduce the associated risk of death. This is the largest study to date, comprising 123 cases, demonstrating the effectiveness and feasibility of TIPS in patients with HCC and proposing a post-TIPS prognostic evaluation model.

The median OS in our study of 10.7 (range, 1.1-55.2) months, 10.3 (range, 1.1-51.3) months in patients with RA, and 10.8 (range, 1.2-55.2) months in patients with variceal bleeding is in disagreement with previous studies showing a 6-month median OS of RA in end-stage liver disease [3]. Liu et al. reported 77 days as a median OS post-TIPS in HCC with SPH [22]. The survival of these enrolled patients, considering tumor factors, was better than that in previous studies, especially in BCLC classification C (median 7.8 months) and D (median
5.3 months) $[3,13,17,18,22,23]$. This may be related to the following: (1) Three months post-TIPS, the RA control was $90.5 \%$ (Fig. 3), and only $1.4 \%(1 / 72)$ of patients had re-bleeding; (2) 41 (33.3\%) patients had downgraded $\mathrm{C}-\mathrm{P}$ staging and greatly improved liver function; 3) 73 (59.3\%) patients received intrahepatic local treatment, which may have reduced the liver tumor burden.

Child-Pugh staging is one of the most widely used liver function assessments, but its appropriateness for use in HCC is unclear, especially in patients with SPH $[15,24$, 25]. Ascites and hypoalbuminemia may be caused by $\mathrm{SPH}$. A different liver function evaluation is needed for these patients post-TIPS. By comparing the five indicators of Child-Pugh staging pre-TIPS and one month post-TIPS, we found that PT, bilirubin, and HE had increased and that 41 (33.3\%) patients had a lowered C-P stage post-TIPS. The post-TIPS albumin level increased slightly (median: 34.7 versus $33.2 \mathrm{~g} / \mathrm{l})(P=0.134)$. PostTIPS grade $2 / 3$ ascites was reduced from $54.5 \%$ to $13.0 \%(P<0.001)$. Therefore, improvement of albumin and ascites improved the $\mathrm{C}-\mathrm{P}$ stage. Our study further used five indicators of $\mathrm{C}-\mathrm{P}$ stage to explore the impact of TIPS on OS. The univariate analysis and multivariate analysis found differences in PT, bilirubin, and grade of ascites. The Cox proportional-hazards regression analysis showed that lower post-TIPS grade of ascites 


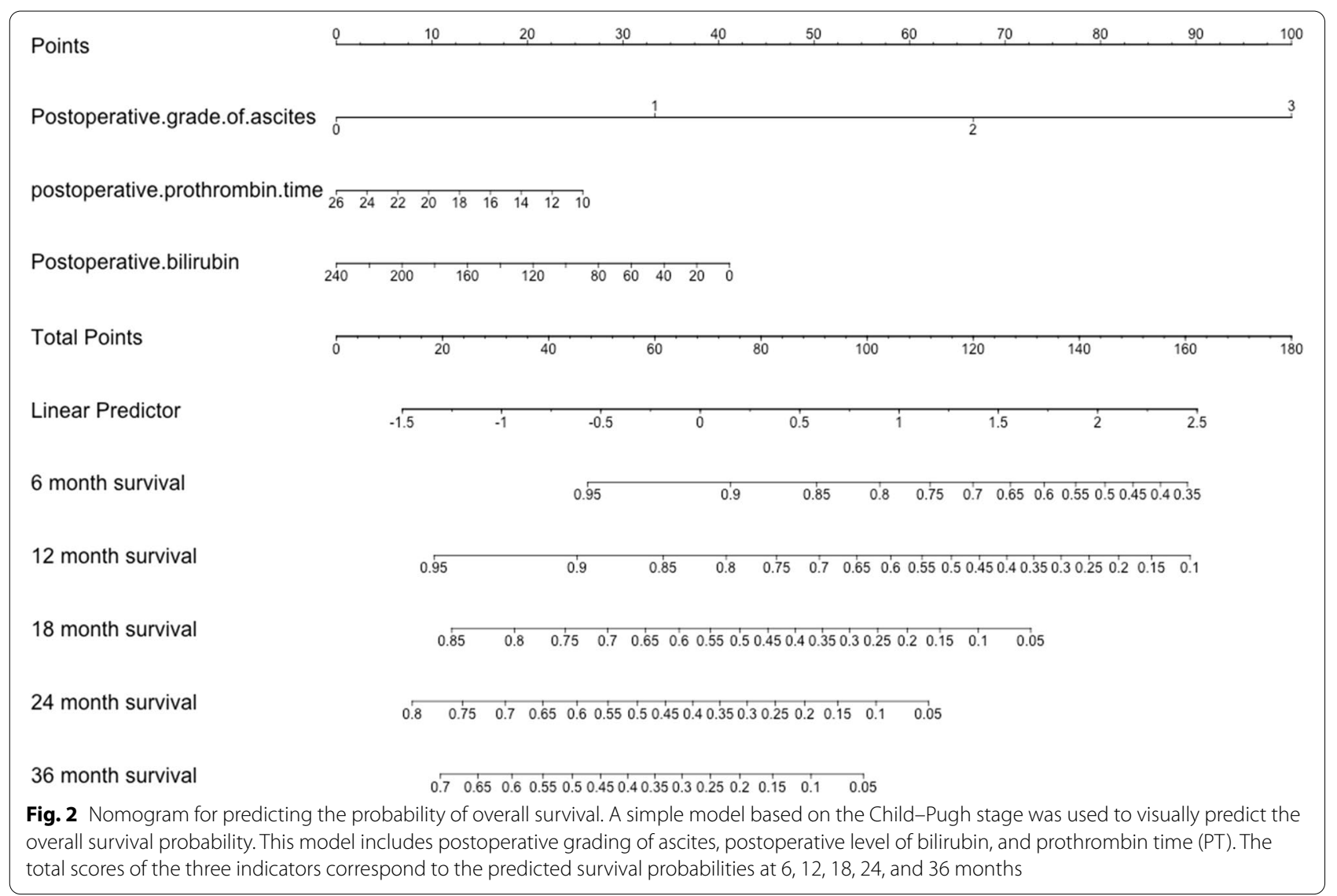

[(0/1)/(2/3): $P=0.014]$, lower post-TIPS level of bilirubin $[<34 / \geq 34 \mu \mathrm{mol} / \mathrm{L}: P=0.022]$, and post-TIPS PT prolonged $<6$ s $[<6 / \geq 6$ s: $P=0.001]$ were independent predictors of better OS. There was no difference in albumin level $[<35 / \geq 35 \mathrm{~g} / \mathrm{L}: P=0.846]$, possibly because hypoalbuminemia and ascites are interrelated indicators and influence one another, and that albumin supplementation therapy may lead to an increase in albumin levels (and thus, an improvement in C-P stage), but may not accurately reflect the liver's ability to synthesize albumin. Based on the above shortcomings, we developed the nomogram prediction system comprising the three indicators of post-TIPS, PT, bilirubin, and grade of ascites to replace the $\mathrm{C}-\mathrm{P}$ system. This model uses grading to reflect the true liver function in $\mathrm{HCC}$ with $\mathrm{SPH}$.

There has been no guideline for the management of HCC with SPH. SPH affects HCC treatment strategy, and HCC makes the treatment of SPH more conservative. Therefore, our research provided some data for the management of these patients. Our research also reflected that a better C-P score after TIPS seems to result in a longer OS. We explained this result on the basis of the improvement of liver function and subsequent antitumor therapy activity. Therefore, the question arises whether earlier TIPS may bring survival benefits for HCC with $\mathrm{SPH}$, just as early TIPS for high-risk variceal bleeding obviously improves the 6-week re-bleeding rate and 1-year survival compared with endoscopic treatment [26]. One key reason is that early TIPS reduces the impact of re-bleeding on the liver function of patients with endstage liver disease [27]. However, in HCC patients with $\mathrm{SPH}$, liver function suffers from bleeding and refractory ascites, which severely affects the prognosis and also prevents some patients from entering the subsequent antitumor treatment. Our study showed a more aggressive TIPS strategy in HCC with SPH to improve liver function so that HCC patients can tolerate subsequent antitumor treatments and obtain survival benefits. This benefit also needs to be further confirmed by more large-scale prospective studies.

Our study also explored the impact of tumor burden factors on OS. Good OS was associated with a lower BCLC classification $(P<0.001)$, no PVTT $(P<0.001)$, a single tumor $(P=0.029)$, a lower level of AFP $(P<0.001)$, and intrahepatic local treatment $(P<0.001)$. AFP is an independent prognostic factor, and the prognostic factors identified are similar to those identified in previous studies. We showed that the prognosis of HCC with SPH 

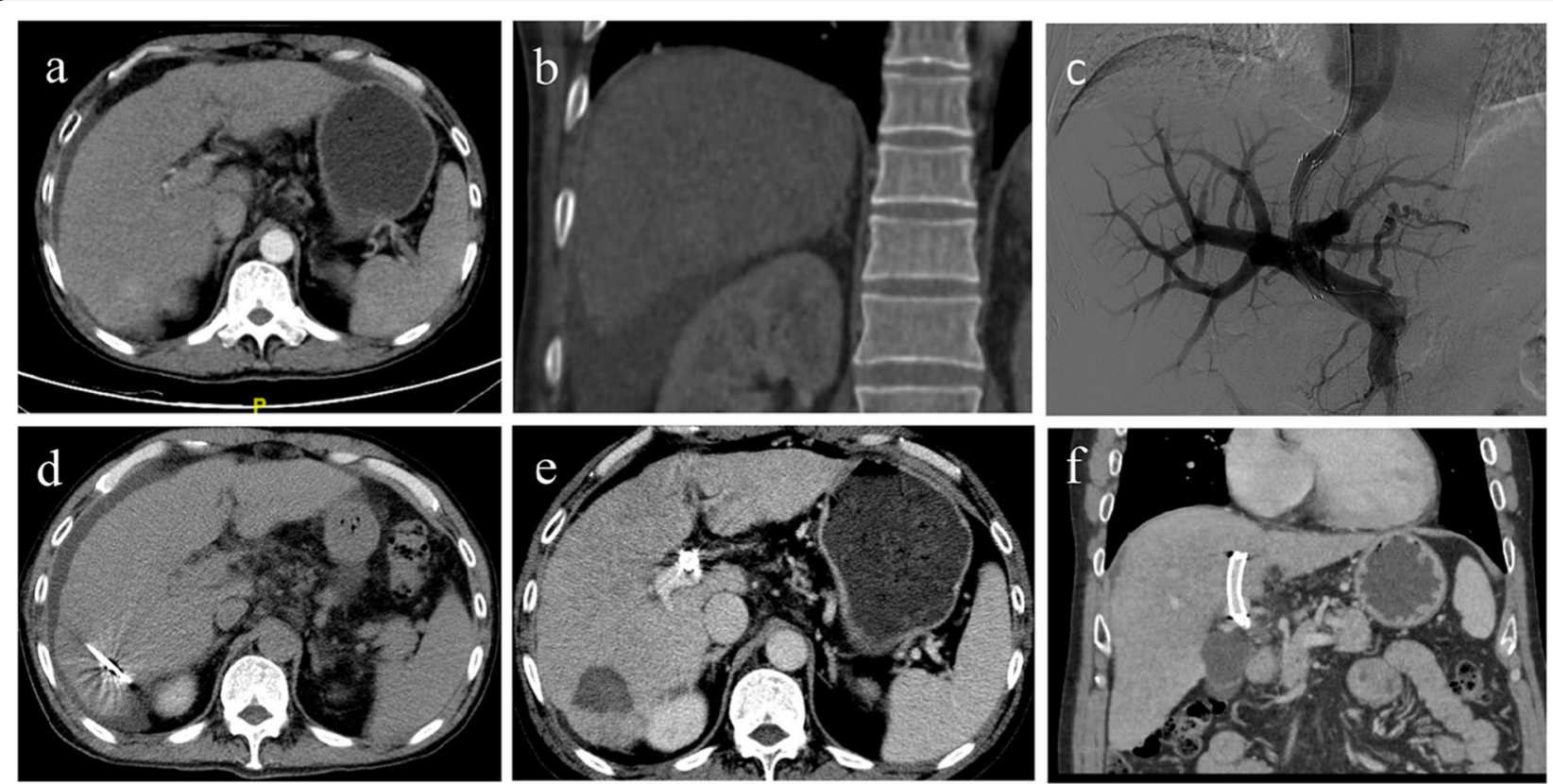

Fig. 3 Case of TIPS. A 53-year-old male patient with a primary hepatocellular carcinoma (HCC) with a diameter of $3 \mathrm{~cm}$. He had experienced a repeated diuretic therapy because of his refractory ascites. a, b Ascites and tumor before transjugular intrahepatic portosystemic shunt (TIPS). The Child-Pugh score and Child-Pugh stage were 9 and (B), respectively. c TIPS was completed. d Two weeks after TIPS, a microwave ablation treatment was implemented. e, f One month after TIPS, the reevaluated Child-Pugh score and Child-Pugh stage were 7 and (B) respectively. The overall survival of the patient exceeded four years, and he is still alive

was determined by multiple factors, including tumor burden [15, 28-30].

Our study showed a technical success rate of $98.4 \%$ (121/123). Two patients (1.6\%) achieved success for the second time, which was a higher result than that reported in a previous study [31]. Although $65 \%$ of the patients in our study had PVTT, most of the patients with tumor thrombus did not have secondary portal cavernous evolution. Technically, it is not too difficult compared to regular TIPS, and only three patients with severe portal vein cavernosis required percutaneous liver puncture and venography to complete TIPS. Although PVTT is a relative contraindication for TIPS, with the emergence of new stent-grafts, the long-term shunt patency in patients with PVTT can be improved. Moreover, TIPS can quickly relieve the symptoms of portal hypertension caused by PVTT, improve opportunities for subsequent targeted therapy, and also help control tumor thrombus. This treatment modality deserves further exploration of its benefits in patients with PVTT. One $(0.8 \%)$ patient required interventional embolization for intraabdominal hemorrhage. Our study identified fewer adverse events than the study by Liu et al., [22] who reported the incidence of tumor rupture of $8.6 \%$ in 58 patients, which may have been related to PVTT in all of their patients. The incidence of HE in our study was $10.6 \%$, lower than the rate of $44 \%$ reported by Wallace et al., [32] which may be related to the $8 \mathrm{~mm}$ diameter stents we used.

Our research has a few limitations. The median followup time of patients with the BCLC classifications of A and B was only 12.5 months, and $50 \%$ of these patients were still living, thus reducing the OS of these patients. The nomogram prediction based on retrospective research and a sample of 123 cases requires more cases to verify our results.

\section{Conclusion}

TIPS is safe and effective for HCC with SPH. This procedure can relieve the symptoms, enable subsequent antitumor therapy, and bring survival benefits, which may come from the improved liver function from the reduced C-P stage.

\section{Abbreviations \\ HCC: Hepatocellular carcinoma; SPH: Symptomatic portal hypertension; TIPS: Transjugular intrahepatic portosystemic shunt; C-P: Child-Pugh; RA: Refrac- tory ascites; PPG: Portosystemic pressure gradient; CDUS: Color doppler ultra- sonography; CR: Complete response; PR: Partial response; NR: None response; TACE: Transarterial chemoembolization; MWA: Microwave ablation; OS: Overall survival; AFP: Alpha-fetoprotein; PVTT: Portal vein tumor thrombosis; MELD: Model of end-stage liver disease; IQR: Interquartile range.}

\section{Acknowledgements}

We thank the patients enrolled in this study. 


\section{Authors' contributions}

Conceptualization: Fei Gao, Data curation: Zhenkang Qiu, Zhanwang Xiang, Formal analysis:Zhanwang Xiang, Kai Feng, Investigation: Huzheng Yan, Kai Feng, Zhanwang Xiang, Methodology: Huzheng Yan, Fei Gao, Project administration: Fei Gao, Mingsheng Huang, Resources: Fei Gao, Mingsheng Huang, Software: Huzheng Yan, Supervision: Fei Gao, Writing - original draft: Huzheng Yan, Writing - review \& editing: Fei Gao, Mingsheng Huang. The author(s) read and approved the final manuscript.

\section{Funding}

This work was supported by Sanming Project of Medicine in Shenzhen (fund No:SZSM201612053).

\section{Availability of data and materials}

The datasets used and/or analysed during the current study are available from the corresponding author on reasonable request.

\section{Declarations}

\section{Ethics approval and consent to participate}

The ethics committee of the Sun Yat-sen University Cancer Center approved the study and waived the requirement for informed consent because of its low risk.

\section{Consent for publication}

This research has been approved by all authors for publication.

\section{Competing interests}

We declare no competing interests.

\begin{abstract}
Author details
'Department of Interventional Radiology, The Third Affiliated Hospital of Sun Yat-Sen University, 600 Tianhe Road, Guangzhou 510630, China. ${ }^{2}$ Department of Minimally Invasive \& Interventional Radiology, State Key Laboratory of Oncology in South China, Collaborative Innovation Center for Cancer Medicine, Sun Yat-Sen University Cancer Center, 651 Dongfeng Road East, Guangzhou 510060, China. ${ }^{3}$ Department of Radiology, The Third People's Hospital of Shenzhen, Shenzhen, China.
\end{abstract}

Received: 13 September 2021 Accepted: 5 February 2022 Published online: 19 February 2022

\section{References}

1. Bray F, Ferlay J, Soerjomataram I. Global cancer statistics 2018: GLOBOCAN estimates of incidence and mortality worldwide for 36 cancers in 185 countries. CA: A Cancer Journal for Clinicians; 2018.

2. European Association For The Study Of The Liver, European Organisation For Research And Treatment Of Cancer. EASL-EORTC clinical practice guidelines: management of hepatocellular carcinoma. J Hepatol. 2012;56:908-43.

3. Angeli P, Bernardi M , Villanueva, Càndid, et al. European Association for the Study of the Liver, EASL Clinical Practice Guidelines for the management of patients with decompensated cirrhosis. J Hepatol. 2018;69:406-60.

4. de Franchis R, Baveno VI Faculty. Expanding consensus in portal hypertension: Report of the Baveno VI Consensus Workshop: Stratifying risk and individualizing care for portal hypertension. J Hepatol. 2015;63:743-52.

5. Berzigotti A, Seijo S, Arena U, et al. Elastography, spleen size, and platelet count identify portal hypertension in patients with compensated cirrhosis. Gastroenterology. 2013;144:102-111.e1.

6. Llop E, Berzigotti A, Reig M, et al. Assessment of portal hypertension by transient elastography in patients with compensated cirrhosis and potentially resectable liver tumors. J Hepatol. 2012;56:103-8.

7. Doyle A, Gorgen A, Muaddi H, et al. Outcomes of radiofrequency ablation as first-line therapy for hepatocellular carcinoma less than $3 \mathrm{~cm}$ in potentially transplantable patients. J Hepatol. 2019;70:866-73.
8. Y Zhao Y-N Zhang K-T Wang et al 2020 Lenvatinib for hepatocellular carcinoma: From preclinical mechanisms to anti-cancer therapy Biochim Biophys Acta Rev Cancer undefined 188391

9. Bouattour M, Mehta N, He AR, et al. Systemic Treatment for Advanced Hepatocellular Carcinoma. Liver Cancer. 2019;8:341-58.

10. Berzigotti A, Reig M, Abraldes JG, et al. Portal hypertension and the outcome of surgery for hepatocellular carcinoma in compensated cirrhosis: A systematic review and meta-analysis. Hepatology. 2015;61:526-36.

11. European Association for the Study of the Liver. EASL Clinical Practice Guidelines: Management of hepatocellular carcinoma. J Hepatol. 2018;69:182-236

12. Bruix J, Sherman M. Management of hepatocellular carcinoma: an update. Hepatology. 2011;53:1020-2.

13. Guadalupe G-T, Abraldes JG, Berzigotti A, et al. Portal hypertensive bleeding in cirrhosis: Risk stratification, diagnosis, and management: 2016 practice guidance by the American Association for the study of liver diseases[J]. Hepatology. 2017;65;310-35.

14 Trebicka J, Bastgen D, Byrtus J, et al. Smaller-Diameter Covered Transjugular Intrahepatic Portosystemic Shunt Stents Are Associated With Increased Survival. Clin Gastroenterol Hepatol. 2019;17:2793-2799.e1.

15 Johnson PJ, Berhane S, Kagebayashi C, et al. Assessment of liver function in patients with hepatocellular carcinoma: a new evidence-based approach-the ALBI grade. J Clin Oncol. 2015;33:550-8.

16 Bettinger D, Knüppel E, Euringer W, et al. Efficacy and safety of transjugular intrahepatic portosystemic shunt (TIPSS) in 40 patients with hepatocellular carcinoma. Aliment Pharmacol Ther. 2015;41:126-36.

17. Liu L, Zhao Y, Qi X, et al. Transjugular intrahepatic portosystemic shunt for symptomatic portal hypertension in hepatocellular carcinoma with portal vein tumor thrombosis. Hepatol Res. 2014;44:621-30.

18 De Santis A, legri C, Kondili L, et al. Hepatocellular carcinoma in cirrhotic patients with transjugular intrahepatic portosystemic shunt: a retrospective case-control study. Dig Liver Dis. 2014;46:726-30.

19 Yamamoto M, Katagiri S, Ariizumi S, et al. Tips for anatomical hepatectomy for hepatocellular carcinoma by the Glissonean pedicle approach (with videos). J Hepatobiliary Pancreat Sci. 2014;21:E53-6.

20. Thabut D, Rudler M, Dib N, et al. Multicenter prospective validation of the Baveno IV and Baveno I//III criteria in cirrhosis patients with variceal bleeding. Hepatology. 2015;61:1024-32.

21. Tandon P, Abraldes JG, Keough A, et al. Risk of Bacterial Infection in Patients With Cirrhosis and Acute Variceal Hemorrhage, Based on Child-Pugh Class, and Effects of Antibiotics. Clin Gastroenterol Hepatol. 2015;13:1189-96.e2.

22. Qiu B, Li K, Dong X, et al. Transjugular Intrahepatic Portosystemic Shunt for Portal Hypertension in Hepatocellular Carcinoma with Portal Vein Tumor Thrombus. Cardiovasc Intervent Radiol. 2017:40:1372-82.

23. Luo $\mathrm{S}-\mathrm{H}$, Chu J-G, Huang $\mathrm{H}$, et al. Safety and efficacy of transjugular intrahepatic portosystemic shunt combined with palliative treatment in patients with hepatocellular carcinoma. World J Clin Cases. 2019;7:1599-610.

24. Yan-Yan W, Xiang B-D, Liang MA, et al. Development and Validation of a Nomogram to Preoperatively Estimate Post-hepatectomy Liver Dysfunction Risk and Long-term Survival in Patients With Hepatocellular Carcinoma.[J]. Ann Surg. 2021;274:e1209-e1217.

25 Hiraoka A, Michitaka K, Kumada T, et al. Prediction of Prognosis of Intermediate-Stage HCC Patients: Validation of the Tumor Marker Score in a Nationwide Database in Japan. Liver Cancer. 2019;8:403-11.

26. LVY, Yang Z, Liu L, et al. Early TIPS with covered stents versus standard treatment for acute variceal bleeding in patients with advanced cirrhosis: a randomised controlled trial. Lancet Gastroenterol Hepatol. 2019;4:587-98.

27 Economopoulos Konstantinos P, Choussein S, SergentanisTheodoros N. Early use of TIPS for cirrhosis and variceal bleeding. N Engl J Med. 2010;363:1375-6 (Early use of TIPS for cirrhosis and variceal bleeding).

28. Zhang S, Liu Y, Chen J, et al. Autoantibody signature in hepatocellular carcinoma using seromics. J Hematol Oncol. 2020;13:85.

29. Chen Zhen-Hua,Zhang Xiu-Ping,Lu Yu-Gang et al. Actual long-term survival in HCC patients with portal vein tumor thrombus after liver resection: a nationwide study.[J] .Hepatol Int, 2020, undefined: undefined. 
30. Zhang X-P, Wang K, Gao Y-Z, et al. Prognostic model for identifying candidates for hepatectomy among patients with hepatocellular carcinoma and hepatic vein invasion. Br J Surg. 2020;107:865-77.

31. Zhao J-B, Feng C, Zhu Q-H, et al. Transjugular intrahepatic portosystemic shunt with covered stents for hepatocellular carcinoma with portal vein tumor thrombosis. World J Gastroenterol. 2014;20:1602-7.

32 Wallace MJ, Madoff DC, Ahrar K, et al. Transjugular intrahepatic portosystemic shunts: experience in the oncology setting. Cancer. 2004;101:337-45

\section{Publisher's Note}

Springer Nature remains neutral with regard to jurisdictional claims in published maps and institutional affiliations.

- fast, convenient online submission

- thorough peer review by experienced researchers in your field

- rapid publication on acceptance

- support for research data, including large and complex data types

- gold Open Access which fosters wider collaboration and increased citations

- maximum visibility for your research: over $100 \mathrm{M}$ website views per year

At BMC, research is always in progress.

Learn more biomedcentral.com/submissions 